\title{
Urgences
}

\section{Le Noroît : vingt ans de souffle}

Entrevue avec Célyne Fortin et René Bonenfant

\section{Paul Chanel Malenfant et René Bonenfant}

Numéro 33, octobre 1991

Poésies parallèles : France - Québec

URI : https://id.erudit.org/iderudit/025674ar

DOI : https://doi.org/10.7202/025674ar

Aller au sommaire du numéro

Éditeur(s)

Urgences

ISSN

0226-9554 (imprimé)

1927-3924 (numérique)

Découvrir la revue

Citer ce document

Malenfant, P. C. \& Bonenfant, R. (1991). Le Noroît : vingt ans de souffle :

entrevue avec Célyne Fortin et René Bonenfant. Urgences, (33).

https://doi.org/10.7202/025674ar d'utilisation que vous pouvez consulter en ligne.

https://apropos.erudit.org/fr/usagers/politique-dutilisation/ 


\section{DOCUMENTS}

\section{Le Noroît: vingt ans de souffle}

\section{une entrevue de Paul Chanel Malenfant}

PCM - Quelles sont les raisons et les circonstances qui vous ont incités a fonder les Éditions du Noroît?

RB - En 1971, alors que j'étais déjà dans l'édition universitaire, Célyne et moi avions le projet commun de créer notre propre maison. Comme nous voulions œuvrer dans un champ autre que l'édition commerciale, nous avons pensé à la poésie. Heureux hasard, un bon ami et collègue de travail, Alexis Lefrançois, nous a alors proposé un manuscrit qu'il avait l'intention de publier mais sans avoir arrêté son choix sur un éditeur en particulier et uniquement pour que nous en discutions amicalement avec lui. Nous avons lu son texte, Calcaires, que nous avons trouvé très beau. C'est ainsi, qu'en 1971, l'idée de fonder les Éditions du Noroît a pris spontanément corps dans une coïncidence amicale et dans un manuscrit. Nous avions toujours souhaité assortir la poésie d'un support visuel; dès ce premier livre, nous avons donc voulu engager une concertation entre les poèmes de Lefrançois et les dessins de Miljenko Horvat, qui était aussi un collègue et un ami. Le point de départ des Éditions du Noroît, c'est donc ce petit groupe d'amis qui travaillaient à l'université; c'est aussi l'événement de ce premier livre, Calcaires, dont les textes et les dessins nous ont séduits et qui nous paraissent encore avoir bien résisté au temps.

PCM - Comment vous est venu le choix de ce nom, Le Noroit, pour la maison?

CF - Je viens du nord-ouest de la province, de l'Abitibi. Mon père employait souvent ce mot du vieux français, *Noroît *, qui signifie *vent du nord-ouest *. Nos ancêtres étaient des voyageurs et des marins, j'entendais aussi les gens parler du *suroît», pour désigner le *vent du sud-ouest *. Comme le Noroît est un vent, René et moi espérions, symboliquement, y puiser un nouveau souffle, un souffle de poésie.

PCM - Vous avez choisi de publier exclusivement de la poésie, malgré la réception limitée de ce genre sur le marché littéraire? 
RB - Nous savions d'abord qu'en choisissant la poésie, genre pour lequel les maisons d'édition en général manifestent peu d'enthousiasme, je ne risquais pas d'entrer en conflit d'intérêt avec mon employeur dans l'édition universitaire. En outre, Célyne et moi voulions exprimer notre créativité, explorer des formes nouvelles. L'édition poétique, à l'instar de celle du livre pour enfants, permet cette liberté créatrice, elle stimule l'invention et le renouvellement. Le livre de poèmes doit être bien fait parce qu'il est fait pour durer. Il n'appartient pas à la consommation rapide ou éphémère. En plus d'être relativement peu couvert, le champ de la poésie allait nous permettre d'exercer à chaque nouveau livre notre imagination et notre sens critique.

PCM - En vingt ans, vous avez dâ lire des centaines de manuscrits et vous avez toujours été très discrets, même dans l'amitié que vous entreteniez avec des auteurs, sur les critères esthetiques, théoriques ou poétiques qui présidaient à vos choix. Vous evoquiez parfois le passage des manuscrits sur votre table de chevet... Pourriez-vous maintenant préciser vos critères de lecture?

CF - Notre seul critère, et fondamental, était d'aimer un texte. En ce sens, nous avons souvent refusé le manuscrit d'un «nouvel auteur" qui ne nous plaisait pas, comme nous avons parfois demandé à des auteurs de la maison, et que nous aimions, de retravailler leurs textes. Il nous faudrait donc définir ce que nous aimons? À regarder le large éventail des publications du Noroît, on discerne que des œuvres très diverses nous ont plu. On nous a parfois reproché d'avoir mis en présence des auteurs aussi différents, par exemple, que Jacques Brault, Claude Beausoleil, Denise Desautels et JeanNoël Pontbriand. La lecture d'un manuscrit devait nous lais ser une résonance, cette musique personnelle à chacun et qui persiste dans la mémoire au delà de la lecture. Nous étions très sensibles à ces mots qui continuent de nous habiter, à ces rythmes qui durent après les poèmes, et qui sont aussi la poésie.

RB - Nous avons choisi de publier des livres qui nous plaisaient et nous avons toujours soutenu notre devise: *Le Noroît souffle où il veut *. Pendant ces vingt années, nous croyions que c'était aussi notre privilège, en quelque sorte, de 
miser sur des textes susceptibles de rejoindre un public plus large. Certes, nos choix ont été * subjectifs * mais nous pensons que la subjectivité est la seule manière de s'engager véritablement en poésie.

CF - Nous avons lu de très nombreux manuscrits et au fur et à mesure de ces lectures nous avons appris à discerner ce que nous aimions vraiment. Même la lecture des manuscrits médiocres fait partie de cet apprentissage. À travers tous ces textes que nous avons reçus, j'ai toujours reconnu la nécessité d'* être de son temps *; notre époque ne peut pas réinventer la rime. Les thèmes poétiques, eux, sont toujours les mêmes, l'amour, la mort..., mais ils sont constamment réévalués à la faveur des événements contemporains. Une forme passéiste - et nous avons reçu un nombre prodigieux de manuscrits, écrits dans des formes archaïques par de très jeunes auteurs - ne peut pas rejoindre le public actuel. L'enseignement scolaire contribue peut-être à présenter une vision très conventionnelle, très traditionnelle, des formes de la poésie?

RB - Un autre critère, celui-ci sans doute encore plus difficile à définir, présidait à nos choix. Nous voulions encourager de nouveaux auteurs, garder une oreille attentive à des textes peut-être inachevés mais prometteurs. Nous nous disions qu'il faut un premier recueil pour engager une œuvre et que la publication stimule aussi le travail. En ce sens, nous n'avons pas publié que des chefs-d'œuvre, mais nous avons été assez rarement déçus par les promesses ou les lendemains des textes auxquels nous avons fait confiance.

PCM - Croyez-vous avoir été des lecteurs autodidactes de poésie? votre lecture s'appuyait-elle aussi sur une connaissance des questions de "poétique", sur des propositions plus theoriques?

CF - Je suis autodidacte.

RB - Je me considère aussi surtout comme un autodidacte. J'ai, bien sûr, pris connaissance de quelques théories; on sait qu'elles s'épuisent vite... Jai donc toujours préféré maintenir un rapport très concret avec notre travail. En s'engageant à publier des manuscrits, on consent à consacrer des heures à la préparation d'un texte, à voir à toutes les étapes de la fabrication matérielle du livre et... à payer des factures! Si nous avions consacré tout notre temps aux théories littéraires 
susceptibles d'éclairer nos choix, nous aurions manqué d'énergie pour ce qui nous tenait à cœur, à savoir faire des livres. Nous laissions ainsi à d'autres l'étude des racines, des sources ou des appartenances théoriques.

PCM - Vous reconnaissez que la production du Noroit se caracterise par son éclectisme ou encore par son hospitalité a une très grande diversité de voix; discernez-vous dans votre production de vingt ans, un fil conducteur, une orientation litteraire ou une quelconque ligne directrice?

RB - Nous avons sans doute été plus sensibles aux textes * lisibles *, à ces textes qui tout en proposant une expérience d'écriture, voire en travaillant à l'éclatement des genres, demeurent accessibles. Par personnalité et par goût, nous sommes restés plus distants à l'égard d'un certain hermétisme même si nous reconnaissons l'intérêt des recherches et des formes plus abstraites. Nous n'avons jamais prétendu être à la fine pointe de l'expérimentation mais nous avons la certitude d'avoir publié des livres d'une bonne qualité littéraire.

CF - Les éditions du Norôit ne se sont jamais posées en * école*; par ailleurs, la critique a déjà assez justement parlé à notre propos de *nouveau lyrisme*, une formule qui parvient à nous désigner sans trop nous définir ou nous limiter.

RB - Je ne sais pas dans quelle mesure des poètes aussi différents que Amyot, Beausoleil - je procède par ordre alphabétique -, Brault, Charlebois, Laberge, Pontbriand, Uguay... pourraient se retrouver sous le couvert du * nouveau lyrisme *... L'œuvre de chaque auteur résiste, jusqu'à un certain point, à toute étiquette.

PCM - Considérez-vous avoir surtout publié des auteurs ou des textes aux éditions du Noroît, des auteurs ou des poèmes?

CF - Nous avons d'abord voulu publier des auteurs. L'œuvre d'un poète ne tient pas à un seul livre et nous avons toujours misé sur la continuité du travail de nos auteurs, sur la succession de leurs livres. Si nous n'avions publié que des textes, il nous aurait été indifférent de publier un seul recueil d'un auteur.

RB - C'est bien la présence manifeste des auteurs dans leurs textes qui nous intéressait. 
PCM - Quels seraient les auteurs ou les textes dont vous vous sentez, a titre de "simples lecteurs" et, si possible, abstraction faite de votre rôle d'éditeurs, le plus près?

$\mathbf{R B}-$ En vingt ans, nous avons publié plus de deux cents recueils et je me sens sincèrement très proche de tous ces livres. Bien sûr, au fil des années, j'ai pris de la distance en regard de certains d'entre eux où nous avions cru déceler un germe, une amorce, et qui n'ont pas tenu la promesse de la continuité. L'ensemble des recueils du Noroît me parlent tout de même beaucoup. Certains livres, tels qu'en eux-mêmes, ne sont pas toujours "représentatifs" mais ils m'importent quand ils constituent des étapes importantes dans l'évolution du travail d'un poète.

PCM - Vous ne devez garder, sur votre table de chevet, que trois livres choisis parmi toute la production du Noroit?

RB - Trois livres? J'hésite vraiment beaucoup... Je garderais sûrement Calcaires de Lefrançois, parce que c'est notre premier livre, parce que l'auteur est un ami et que son œuvre importante est malheureusement méconnue. Pour d'autres raisons, qui sont aussi des raisons émotives, je retiendrais les livres de Marie Uguay et de Michel Beaulieu, deux cuvres considérables, trop tôt interrompues. Mais non loin de ma table de chevet, je conserverais tous les livres du Noroît...

CF - Je choisirais les livres de trois auteurs relativement peu connus et que j'aime beaucoup: Rachel Leclerc, Michel Savard et Jean Chapdelaine Gagnon. Pour cette petite musique persistante que j'évoquais... une très belle musique... Leurs livres n'ont pas vraiment émergé de l'ensemble de la production. Ces poètes ne sont peut-être pas modernes ou *à la mode , ils sont contemporains. Ils savent parler du monde d'aujourd'hui dans des mots actuels. Je regrette qu'ils ne soient pas mieux connus et appréciés.

PCM - La qualité matérielle des livres du Noroît a toujours exprimé votre intérêt conjoint pour la poesie et pour les arts visuels en général; d'où vous vient ce goût pour l'association du poétique et du visuel, ce goût pour les beaux livres?

CF - Entre nous, nous nous disions que René était le * littéraire * et que j'étais la *visuelle *... Lorsque nous avons projeté de créer une maison d'édition, nous voulions nous 
faire plaisir à l'un et à l'autre. En ce sens, la conception et la réalisation des beaux livres et des livres d'artistes conciliaient nos goûts respectifs. De plus nous voulions donner aux gens le goût de lire de la poésie en la leur présentant dans de beaux objets qu'on a plaisir à regarder, à reprendre.

RB - Puisqu'il était convenu que le Noroît ne deviendrait pas notre gagne-pain, nous voulions que ce travail d'éditeur nous procure le plaisir des typographies soignées, des papiers variés, des belles matières. Nous voulions aussi que le goût et la personnalité des auteurs puissent s'exprimer dans la présentation matérielle de leurs livres, dans le choix des couleurs, des mises en pages, des formats. Le plaisir d'éditer tient à tous ces aspects concrets relatifs à la facture même des livres.

CF - Au moment où nous avons commencé à éditer, peu de maisons se préoccupaient vraiment de la présentation matérielle et de la qualité esthétique des livres. À l'exception de Roland Giguère qui avait été un pionnier avec les Éditions Erta. Mais, dans l'édition commerciale, on trouvait peu d'innovation. Si on regarde maintenant la qualité visuelle des livres de Québec/Amérique et de VLB éditeur, entre autres, il me semble que nous avons été des précurseurs en ce domaine et que notre travail a été exemplaire. Nous sommes toujours très fiers de nos livres même quand nous les comparons, à Francfort par exemple, à ceux des grandes maisons d'édition.

PCM - Comment s'est effectuée la répartition des tâches au Noroît? lecture des manuscrits, décision de publier, conception des livres, diffusion, promotion?

RB - Notre personnel peu nombreux n'attendait pas de longues descriptions des tâches ! Célyne et moi avons assumé à tour de rôle ou ensemble toutes les responsabilités. Ainsi, devant un manuscrit qui nous plaisait à tous les deux, après relecture la décision de publier était rapidement prise. Décision contraire devant un texte qui ne plaisait ni à l'un ni à l'autre. Notre travail de lecture a donc toujours été très concerté. En regard de la fabrication matérielle des livres, comme l'édition était déjà mon métier, je suis sans doute intervenu davantage tout au début. Mais Célyne a tout appris de ce métier... sur le tas! Nous nous sommes alors partagé la conduite des divers projets. Par ailleurs, Célyne 
s'est occupée davantage de la gestion, de la comptabilité, des droits d'auteurs, bref, des chiffres! De mon côté, je veillais à la publicité, aux relations avec le distributeur, à la manutention des caisses... Toutes ces tâches s'enchevêtraient dans un joyeux mélange!

PCM - Quel type de relation avez-vous voulu entretenir avec les auteurs du Noroît?

CF - Le Noroît, c'était chez nous, dans notre maison, dans notre salle à dîner, dans notre cuisine, dans notre salon... Nous n'avons jamais eu de bureau officiel et de ce fait, à notre insu peut-être, nous avons créé des liens plus intimes avec les auteurs. Certains auteurs parlaient même de famille; plusieurs sont devenus des amis très proches. Il ne faut pas oublier non plus que le premier auteur du Noroît était un ami... De plus notre manière de travailler nous faisait préférer les relations personnelles aux relations d'affaires. Nous aimions consulter les auteurs sur leurs goûts, sur les artistes avec qui ils aimeraient travailler, sur la vision qu'ils avaient de leurs livres. Lorsque l'UNEQ a désigné les éditeurs comme les patrons des auteurs nous nous sommes sentis mal à l'aise; nous n'avions jamais été des * patrons * pour personne...

$\mathbf{R B}$ - C'est sans doute pourquoi plusieurs recueils ont été publiés au Noroît sans contrat avec les auteurs. Je ne cite pas cette situation en exemple mais elle est révélatrice de notre préférence pour les relations d'amitié et de confiance plutôt que pour les relations d'affaires. Il ne nous est jamais apparu essentiel de définir à l'avance, juridiquement, légalement, ce que devrait être une relation.

CF - C'est Michel Beaulieu qui a rédigé le premier contrat que nous avons utilisé.

RB - Et nous l'avons utilisé pendant plusieurs années jusqu'à ce que nous disposions de celui de l'UNEQ. Il faut préciser que Michel avait été éditeur à l'Estérel où il avait pris des habitudes de précisions légales. Nous lui avons donc demandé de rédiger ce contrat. Il était parti d'un texte très élaboré, celui du contrat «type * de plusieurs maisons d'édition, pour ne retenir finalement qu'une dizaine de clauses essentielles. Nous avons utilisé cette version simplifiée pendant plusieurs années, sans dommage pour nous, ni pour nos auteurs! 
PCM - Quelles sont les raisons qui, croyez-vous, ont incité les poètes a publier au Noroit?

RB - Les auteurs appréciaient notre mode de fonctionnement informel et spontané. La facture matérielle de nos livres constituait aussi un attrait pour eux. À la différence de ce qui se passe dans la plupart des maisons d'édition, les poètes du Noroît aimaient être impliqués dans toutes les étapes de la fabrication de leur livre, porter leur texte non seulement jusqu'à l'état de manuscrit mais jusqu'à l'état de livre. Cette intervention fait partie du processus même de la création poétique puisque le choix des aspects visuels d'un livre de poésie relève déjà d'une proposition ou d'une suggestion de lecture.

CF - Plusieurs poètes appréciaient l'ouverture du Noroît, le fait que nous n'appartenions ni à une école, ni à un mouvement précis, ni à une optique déterminée. Ils se sentaient plus libres au Noroît que dans d'autres maisons plus strictement orientées.

PCM - Ces dernières années, vous avez tenté plusieurs expériences de concertation éditoriale avec des maisons d'edition européennes. Que ressort-il de ces collaborations pour la poésie du Noroit?

RB - En effet, depuis 6 ans environ, nous avons fait plusieurs co-éditions, à titre d'expériences et en demeurant très lucides sur la portée financière limitée de telles opérations. Le marché français est plutôt hermétique aux livres québécois. Nous espérions surtout donner à des poètes d'ici la chance de se faire entendre en France. Les résultats sont inévitablement lents et, de fait, relèvent davantage d'une certaine reconnaissance de quelques auteurs que d'une véritable ouverture du marché pour le Noroît. Nous savons que les éditeurs européens de notre taille connaissent des problèmes de diffusion de leurs auteurs identiques aux nôtres. Qu'ils publient à leur enseigne un poète d'ici n'assure donc en rien à celui-ci la couverture de son livre dans les médias ou les revues de poésie. La co-édition nous semble un placement littéraire à long terme susceptible de rapporter surtout aux auteurs. En ce sens, des poètes comme Claude Beausoleil et Hélène Dorion connaissent maintenant une certaine audience en France. Pour nous, éditeurs, il ne s'agit pas d'une formule d'affaires mais d'une sorte de troc culturel: au lieu de sous ou 
de traites bancaires, nous échangeons des livres. Ces échanges permettent donc aussi à des poètes français de se faire connaître ici. Nous demeurons très réalistes, cependant, quant aux effets de cette circulation des livres sur leur consommation. Tout au début du Noroît, nous faisions un service de presse en France. Nous avons d'ailleurs eu d'excellents articles d'Alain Bosquet dans la $N R F$. Les gens du milieu, làbas, se sont bientôt désolés de lire des commentaires élogieux de la critique... sur des livres qui n'étaient pas disponibles sur le marché! Et la critique, à son tour, s'est désolée de parler de livres introuvables! Nous avons pourtant transporté bien des livres dans nos bagages pour les déposer chez des libraires sympathiques à la cause de la poésie. Mais les libraires sympathiques à la cause ne sont pas toujours les meilleurs hommes d'affaires... Nous avons donc eu l'idée des co-éditions pour qu'un certain nombre d'exemplaires de nos livres publiés à l'enseigne conjointe du Noroît et du Dé bleu, du Noroît et du Castor astral, du Noroît et de Jacques Brémond, soit accessible, en France, au public lecteur.

PCM - Au fil des années quelle a êté votre attitude à l'égard des organismes subventionnaires et comment ceux-ci percevaient-ils votre travail éditorial, votre ténacité a l'égard de la poésie québécoise?

RB - Nous avons toujours été assez bien reçus tant par le ministère des Affaires culturelles du Québec que par le Conseil des arts du Canada. Dans l'ensemble, nous répondions bien aux critères qualitatifs et quantitatifs de leurs différents programmes de subvention. Au cours de ces 20 ans, nous avons donc eu des relations efficaces avec ces organismes. Que nous ayons persévéré d'année en année, que nous ayons maintenu un rythme convenable quant au nombre des titres publiés, quant à la qualité des textes et de leur présentation, témoigne de la bonne nature de ces relations. Le Noroît n'a été ni choyé ni boycotté; mais je crois que les jurys, qui s'appuient sur des normes précises, ont somme toute bien reconnu notre persévérance.

PCM - Comment percevez-vous le travail des autres maisons qui publient la poésie québécoise: les Éditions des Forges, l'Hexagone, les Herbes rouges, entre autres? En quoi le Noroit vous semble-t-il se distinguer de ces autres lieux? 
$\mathbf{R B}$ - Tous les responsables de ces maisons étaient nos collègues et nous ne nous sommes jamais sentis en concurrence avec l'une ou l'autre d'entre elles. Les lieux d'édition ne sont jamais assez nombreux et il n'y a jamais trop de livres. Je crois que les jeunes auteurs devraient aussi créer leurs propres maisons. En vingt ans, le Noroît s'est constitué une bonne équipe d'auteurs et nous voulions être responsables à l'égard de chacun d'eux. En ce sens, nous étions moralement engagés à l'égard d'un poète qui avait déjà publié trois livres chez nous et qui nous proposait son quatrième manuscrit. Il nous était donc devenu plus difficile, même si nous avons toujours voulu donner la chance à de nouvelles voix, de les accueillir. Chaque année, un certain nombre des auteurs du Noroît (entre soixante-quinze et quatre-vingts) nous propose un nouveau manuscrit intéressant: au rythme de publication d'une quinzaine de recueils par an, les places disponibles sont alors rapidement occupées. Maintenant que le Noroît, comme les Forges et les Herbes rouges, ont atteint la maturité, il faudrait que naissent de nouveaux lieux d'écriture, que des auteurs plus jeunes se donnent leurs propres espaces. Nous savons que ce n'est pas facile car les coûts de fabrication des livres ont considérablement augmenté. Calcaires, d'Alexis Lefrançois, ce premier recueil du Noroît tiré à 550 exemplaires, avait coûté approximativement $700 \$$ ou $800 \$$. Pour un livre de cette qualité, il en coûterait maintenant de $3000 \$$ à $4000 \$$. En ce sens, les jeunes qui voudraient se créer un lieu devraient faire preuve d'audace financière.

CF - L'Hexagone a surtout accueilli les auteurs de la génération qui nous précède. Quant aux Éditions des Forges, elles émanent d'abord du milieu universitaire de TroisRivières. Les Herbes rouges ont davantage publié des livres axés sur le formalisme et, de ce fait, elles étaient sans doute plus sélectives dans le choix de leurs auteurs. Le Noroît a toujours été délibérément plus hétérogène, plus ouvert.

RB - Les auteurs restent maintenant toujours libres de publier simultanément dans plusieurs maisons sans vexer l'un ou l'autre éditeur. Claude Beausoleil, par exemple, publie à la fois au Noroît et aux Écrits des Forges, Michel Beaulieu avait déjà publié à l'Hexagone et André Gervais aux Herbes rouges avant de venir chez nous. Je pense qu'il est profitable aux auteurs d'avoir accès à plus d'un milieu éditorial. 
CF - Au moment où on a décidé de vendre le Noroît, je tenais à ce que ce soit la nouvelle génération qui prenne la relève. Les poètes de 30 ans qui sont en train d'écrire leur œuvre vont sans doute renouveler l'identité de la maison.

PCM - Vous discernez donc l'émergence d'une nouvelle génération en poésie québécoise? en quoi ces auvres nouvelles se distingueraient-elles, tant du point de vue du fond que de celui de la forme, des pratiques des aînés que vous avez publiés?

CF - Cette nouvelle génération semble pressée par le temps. Les jeunes poètes veulent publier beaucoup et rapidement. Élise Turcotte, par exemple, publie un livre presque à chaque année. Cette fébrilité contraste avec la lenteur ou la patience d'un Michel Beaulieu qui laissait reposer ses manuscrits 4 à 5 ans avant leur publication.

RB - La question me laisse un peu perplexe... Je crois aussi que les jeunes poètes veulent vraiment et rapidement se faire entendre en poésie. Si on observe l'histoire littéraire, au Québec, on se rend compte que les poètes ont souvent poursuivi diverses activités tout en écrivant de la poésie. Choquette, par exemple, était ambassadeur et on pourrait citer plusieurs poètes qui ont fait une carrière en écrivant leur œuvre. Plusieurs jeunes auteurs s'adonnent à la poésie comme à une activité essentielle, professionnelle en quelque sorte. Au rythme d'un recueil par an, il s'agit vraiment pour eux d'une préoccupation majeure, d'un travail constant.

CF - L'amour reste un thème fondamental, chez les jeunes poètes. Pensons, par exemple, au très beau Où serons-nous dans une heure de Jacques Ouellet que nous avons publié l'an dernier. On observe aussi, dans plusieurs manuscrits qui nous sont soumis, un goût marqué pour le langage cru, pour le vocabulaire ordurier ou scatologique. Une sorte de rage ou de frénésie dans la langue...

RB - Les jeunes poètes me semblent exprimer une plus grande liberté en regard de la forme, sans préméditation, sans se préoccuper de s'inscrire dans une veine déterminée; d'où, chez eux, une certaine fraîcheur et cette spontanéité... Contrairement à ce qui se passait dans les années $60-70$, où les poètes se souciaient de leur appartenance à un mouvement de type «Tel quel* ou autre, par exemple, les jeunes 
émettent plutôt une sorte de cri primal. Rien de fabriqué, chez eux, mais une sorte d'urgence.

PCM - Qu'est-ce qui vous a amené a prendre la décision de quitter le Noroît pour en confier la direction à une nouvelle équipe?

RB - Nous avons assumé un contrat de 20 ans avec le Noroît et, au cours de ces années, nous croyons avoir donné à la maison sa stature et sa personnalité. Avec la maturité, il fallait maintenant lui assurer une relance, un renouvellement et aussi un statut financier moins précaire. En ce sens, si nous avions conservé le Noroît nous aurions sans doute dû, pour le bien de tout le monde et celui de nos auteurs en particulier, nous adjoindre des collaborateurs, nous doter éventuellement d'un bureau et d'une structure juridique plus ferme, bref, faire en sorte que la maison ne repose plus exclusivement sur nos seules épaules. Pour assurer la persistance du Noroît et pour éviter l'essoufflement, nous avons préféré passer le flambeau à d'autres énergies, à une équipe nouvelle pleine d'idées neuves. Ajoutons que la tâche de gestion, avec notre groupe de près de 80 auteurs et de presque autant d'artistes, avec l'accroissement aussi du nombre de titres à notre catalogue, était devenue trop lourde et trop complexe pour deux seules personnes. Il fallait faire la comptabilité, procéder à l'inventaire, bref à tout ce travail technique de plus en plus exigeant et où la créativité a moins cours. Après vingt ans, nous avons finalement souhaité donner un nouveau souffle au Noroît en le confiant à une nouvelle équipe enthousiaste. Enfin, au moment où nous allions prendre cette décision, un poste qui m'intéressait s'est ouvert à Ottawa, j'ai posé ma candidature et je l'ai obtenu. Heureux concours des circonstances. Mais nous séparer du Noroît, un geste déchirant tout de même...

PCM - Quel capital poétique, non seulement en terme de fond, mais en terme de mentalité éditoriale, croyez-vous transmettre a la nouvelle équipe de direction?

RB - Compte tenu de toute l'énergie que nous avons investie au Noroît pendant 20 ans, nous tenions à ce que la maison poursuive avant tout sa vocation littéraire et qu'elle continue d'accorder une place prépondérante à la poésie. C'est pourquoi, quand nous avons cherché une relève, nous avons 
d'abord regardé parmi nos auteurs. Comme nous avions toujours été très près d'eux, nous croyions qu'il s'en trouverait dans le groupe pour assurer au Noroît une succession fidèle à l'esprit que nous lui avions inculqué. Une nouvelle équipe s'est alors constituée avec Hélène Dorion, Paul Bélanger et Claude Prud'Homme; tous ont des goûts et des intérêts littéraires; en outre, ils sont prêts à investir du temps et de l'énergie sans attendre de retombées financières immédiates. Nous ne voulions pas que le Noroît devienne une compagnie ou une institution impersonnelle. L'actuelle équipe connait très bien le mode de fonctionnement qui était le nôtre. Maintenant, le Noroît leur appartient et nous ne voulons pas intervenir dans leurs choix éditoriaux et dans leurs formules de gestion.

CF - Le capital poétique du Noroît, c'est aussi son inscription dans l'histoire littéraire du Québec, dans l'institution et dans le paysage de la poésie d'ici depuis vingt ans. Nous laissons un nom et une maison que les discours sur la poésie ne peuvent plus ignorer... même s'ils l'ont déjà fait.

PCM - Comment, enfin, rêvez-vous la continuité de votre travail par la nouvelle équipe?

CF - Au départ, la nouvelle équipe nous a assurés de sa volonté de continuer à publier de la poésie et dans des livres de belle facture. Tous ses membres s'intéressent aussi aux livres d'artistes. Qui sait s'ils ne pourraient pas éditer un jour d'autres types d'objets poétiques ou du roman? Quoi qu'il en soit, je souhaite que le Noroît demeure toujours un lieu de poésie...

RB - La passation du Noroît à la nouvelle équipe a été très simple et cordiale. Nous souhaitons que le Noroît continue de souffler en poésie... Mais les circonstances peuvent toujours changer et la nouvelle direction est libre. Nous sous sommes aussi entendus pour que les auteurs du Noroît ne soient pas laissés en plan, pour que soit assurée la continuité de leur œuvre. Ce ne sont pas des obligations contractuelles, mais morales et poétiques... 